\title{
Critical Speeding-Up in the Local Dynamics of the Random-Cluster Model
}

\author{
Youjin Deng, ${ }^{1}$ Timothy M. Garoni, ${ }^{1}$ and Alan D. Sokal ${ }^{1,2}$ \\ ${ }^{1}$ Department of Physics, New York University, 4 Washington Place, New York, New York 10003, USA \\ ${ }^{2}$ Department of Mathematics, University College London, London WCIE 6BT, United Kingdom
}

(Received 5 January 2007; published 7 June 2007)

\begin{abstract}
We study the dynamic critical behavior of the local bond-update (Sweeny) dynamics for the FortuinKasteleyn random-cluster model in dimensions $d=2,3$ by Monte Carlo simulation. We show that, for a suitable range of $q$ values, the global observable $\mathcal{S}_{2}$ exhibits "critical speeding-up": it decorrelates well on time scales much less than one sweep. In some cases the dynamic critical exponent for the integrated autocorrelation time is negative. We also show that the dynamic critical exponent $z_{\exp }$ is very close (possibly equal) to the rigorous lower bound $\alpha / \nu$ and quite possibly smaller than the corresponding exponent for the Chayes-Machta-Swendsen-Wang cluster dynamics.
\end{abstract}

DOI: 10.1103/PhysRevLett.98.230602

Dynamic processes in statistical mechanics typically undergo critical slowing-down [1]: the autocorrelation (relaxation) time $\tau$ diverges as the critical point is approached, most often like $\tau \sim \xi^{z}$, where $\xi$ is the spatial correlation length and $z$ is a dynamic critical exponent. In this Letter we would like to draw attention to the converse (and quite unexpected, at least to us) phenomenon of critical speeding-up: some observables $\mathcal{O}$ can exhibit strong decorrelation on time scales much less than one sweep, so that the dominant relaxation modes equilibrate faster (in natural time units) near criticality. As a consequence, the integrated autocorrelation time $\tau_{\text {int, } \mathcal{O}}$ can in some cases tend to zero, so that the dynamic critical exponent $z_{\mathrm{int}, \mathcal{O}}$ is negative. These behaviors also have practical implications for the efficiency of Monte Carlo simulations [2,3] near the critical point.

More precisely, we shall exhibit these phenomena for the Fortuin-Kasteleyn (FK) random-cluster model [4,5] with a local bond-update dynamics [6]. The randomcluster model is a correlated bond-percolation model that is closely related to the Potts spin model $[7,8]$. As such, it plays a major role in the theory of critical phenomena, especially in two dimensions where it arises in recent developments of conformal field theory [9] via its connection with stochastic Loewner evolution (SLE) $[10,11]$.

The random-cluster model with parameters $q, v>0$ is defined on any finite graph $G=(V, E)$ by the partition function

$$
Z=\sum_{A \subseteq E} q^{k(A)} v^{|A|},
$$

where $A$ is the set of "occupied bonds", $|A|$ is the number of occupied bonds, and $k(A)$ is the number of connected components ("clusters") in the graph $(V, A)$. For $q=1$ this reduces to independent bond percolation [12] with occupation probability $p=v /(1+v)$; for integer $q \geq 2$ it provides a graphical representation [4] of the $q$-state ferromagnetic Potts model with nearest-neighbor coupling $J$, where $v=e^{\beta J}-1$. The random-cluster model thus provides an extension of the Potts model that allows all
PACS numbers: 05.50.+q, 05.10.Ln, 05.70.Jk, 64.60.Ht

positive values of $q$, integer or noninteger, to be studied within a unified framework.

The simplest dynamics for the random-cluster model is the local bond-update dynamics first used by Sweeny [6]: choose a bond $e \in E$ at random, erase its current occupation state, and then give it a new occupation state according to the conditional distribution of (1) with the other bonds held fixed [13]. In detail, this means that $e$ will become occupied with probability $v /(1+v)$ [resp. $v /(q+v)$ ] in case the end points of $e$ are (resp. are not) already connected by a path of occupied bonds not using $e$. The efficient implementation of this connectivity check leads to nontrivial algorithmic questions [14] that we will discuss in detail elsewhere [15].

In two dimensions, the behavior of the ferromagnetic Potts/random-cluster model is fairly well understood, thanks to a combination of exact solutions [16], Coulomb-gas methods [17], and conformal field theory [9]. But in dimensions $d \geq 3$, many important aspects remain unclear, including the location of the crossover between second-order and first-order behavior [18], the nature of the critical exponents and their dependence on $q$, the value of the upper critical dimension for noninteger $q$, and the qualitative behavior of the critical curve $v_{c}(q)$ near $q=0$ [19]. Monte Carlo simulations using the Sweeny [6] and Chayes-Machta [20] algorithms will likely play an important role in elucidating these problems.

In this Letter we present the results of Monte Carlo simulations using the Sweeny dynamics on $d$-dimensional simple hypercubic lattices of linear size $L$ with periodic boundary conditions. We shall measure time in units of "hits" of a single bond, but we stress that the natural unit of time is one "sweep" of the lattice, consisting of $d L^{d}$ hits. For any observable $\mathcal{O}$, we define the unnormalized autocorrelation function at time lag $t$,

$$
C_{\mathcal{O}}(t)=\left\langle\mathcal{O}_{s} \mathcal{O}_{s+t}\right\rangle-\langle\mathcal{O}\rangle^{2},
$$

where expectations are taken in the stationary stochastic process (i.e., in equilibrium), and the normalized autocorrelation function $\rho_{\mathcal{O}}(t)=C_{\mathcal{O}}(t) / C_{\mathcal{O}}(0)$. We then define the 


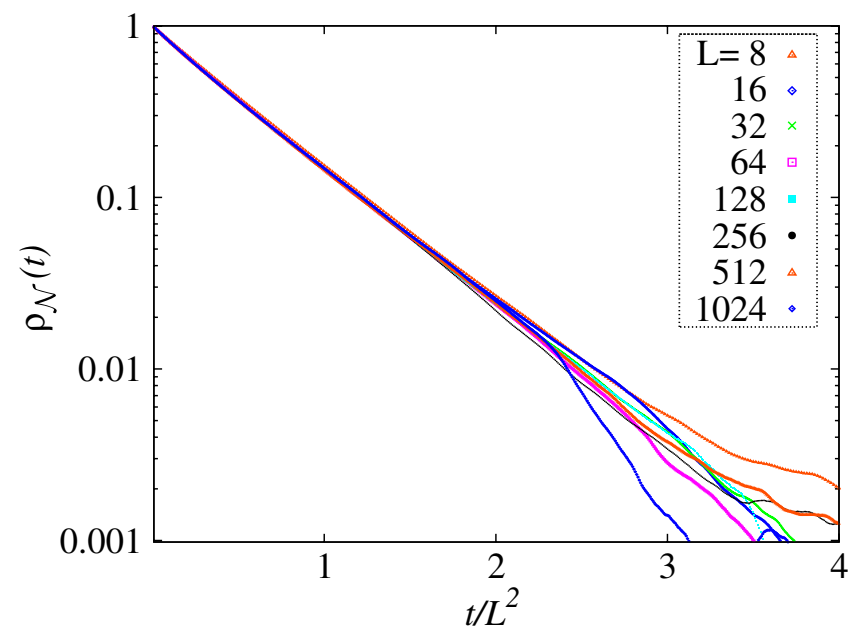

FIG. 1 (color online). Autocorrelation function $\rho_{\mathcal{N}}(t)$ vs $t / L^{2}$ for the critical two-dimensional random-cluster model at $q=$ 0.2 , where time $t$ is measured in hits.

exponential autocorrelation time

$$
\tau_{\exp , \mathcal{O}}=\limsup _{t \rightarrow \pm-\infty} \frac{|t|}{-\log \left|\rho_{\mathcal{O}}(t)\right|}
$$

and the integrated autocorrelation time

$$
\tau_{\mathrm{int}, \mathcal{O}}=\frac{1}{2} \sum_{t=-\infty}^{\infty} \rho_{\mathcal{O}}(t)
$$

Typically all observables $\mathcal{O}$ (except those that, for symmetry reasons, are "orthogonal" to the slowest mode) have the same value $\tau_{\exp , \mathcal{O}}=\tau_{\text {exp }}$. However, they may have very different amplitudes of "overlap" with this slowest mode; in particular, they may have very different values of the integrated autocorrelation time, which controls the efficiency of Monte Carlo simulations [3]. We define dynamic critical exponents $z_{\exp }$ and $z_{\text {int, } \mathcal{O}}$ by $\tau_{\exp } \sim \xi^{\exp }$ and

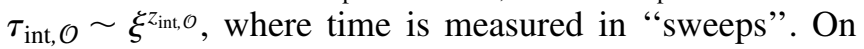
a finite lattice at the critical coupling, $\xi$ can here be replaced by $L$.

In our simulations we measured a variety of observables, among which are the number of occupied bonds $\mathcal{N}=|A|$ and the sum of squares of cluster sizes $\mathcal{S}_{2}=\sum|\mathcal{C}|^{2}$. It is well known [4] that $\left\langle\mathcal{S}_{2}\right\rangle=V \chi$, where $V=L^{d}$ is the volume and $\chi$ is the Potts-model susceptibility. A simple variational argument [21] shows that, in the Sweeny dynamics, $\tau_{\exp } \geq \tau_{\text {int, } \mathcal{N}} \geq$ const $\times C_{H}$, where $C_{H}$ is the specific heat and time is measured in sweeps; hence $z_{\text {exp }} \geq z_{\text {int }, \mathcal{N}} \geq \alpha / \nu$.

We began by performing simulations on the square lattice $(d=2)$ at the exact critical point $v_{c}(q)=\sqrt{q}[16]$ for $q=0.0005,0.005,0.05,0.2,0.5,1,1.5,2,2.5,3,3.5$, and a variety of lattice sizes $4 \leq L \leq 1024$ [22]. In all cases the autocorrelation function of $\mathcal{N}$ is very close to a pure exponential (Fig. 1). The integrated autocorrelation times $\tau_{\text {int, } \mathcal{N}}$ are shown as a function of $q$ and $L$ in Fig. 2, and the corresponding dynamic critical exponents $z_{\exp } \approx$

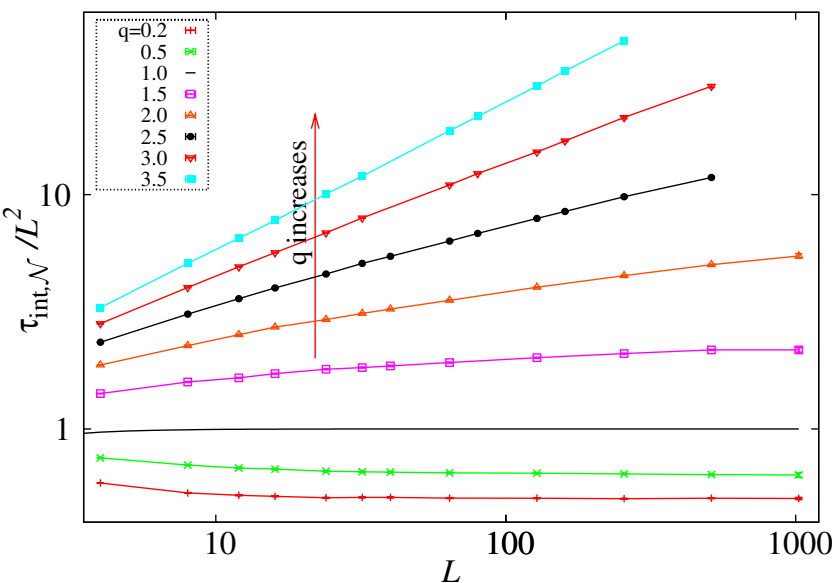

FIG. 2 (color online). Integrated autocorrelation times $\tau_{\text {int, } \mathcal{N}}$ for the critical two-dimensional random-cluster model, as a function of $q$ and $L$. Error bars are in all cases smaller than the plotting symbol. For $q=1$ the analytical result is $\tau_{\text {int, } \mathcal{N}}=$ $\frac{1}{2}\left(d L^{d}-1\right)$.

$z_{\text {int }, \mathcal{N}}$ are shown in Table I. The estimated exponents are only slightly larger than the lower bound $\alpha / \nu$ and could conceivably be equal to it [24]. Perhaps surprisingly, these exponents are slightly smaller than those found recently [25] for the Chayes-Machta-Swendsen-Wang [20] cluster algorithm.

A more interesting and unusual dynamic behavior is exhibited by the observable $\mathcal{S}_{2}$. In Fig. 3 we plot the autocorrelation function $\rho_{\mathcal{S}_{2}}(t)$ for $q=0.2$. Clearly $\rho_{\mathcal{S}_{2}}(t)$ exhibits a fast decay in a time much less than a single sweep (i.e., of order $L^{w}$ for some $w<2$ ) as a prelude to the ultimate exponential decay $\sim e^{-t / \tau_{\text {exp }}}$. To analyze this shorttime behavior, we plot $\rho_{\mathcal{S}_{2}}(t)$ versus $t / L^{w}$ and adjust the exponent $w$ until all the points fall on a scaling curve $\rho_{\mathcal{S}_{2}}(t)=f\left(t / L^{w}\right)$ in the limit $L \rightarrow \infty$. We find $w \approx 0.99$. Furthermore, the function $f$ is very close to $f(x)=(1+$ $a x)^{-r}$ with $a=0.55$ and $r=1.42$ (Fig. 4); in any case it behaves like $f(x) \sim x^{-r}$ as $x \rightarrow \infty$.

TABLE I. Estimated dynamic critical exponents for the twodimensional random-cluster model as a function of $q$. Specificheat exponent $\alpha / \nu$ and red-bond (resp. whole-cluster) fractal dimension $d_{\text {red }}$ (resp. $d_{F}$ ) are shown for comparison [23].

\begin{tabular}{llrccrcc}
\hline \hline \multicolumn{1}{c}{$q$} & \multirow{2}{c}{$z_{\exp }$} & $\alpha / \nu$ & $w$ & $r$ & $z_{\text {int, } \mathcal{S}_{2}}$ & $d_{\text {red }}$ & $d_{F}$ \\
\hline 0.0005 & 0 & -1.9576 & 0.77 & 4.83 & -1.23 & 1.2376 & 1.9965 \\
0.005 & 0 & -1.8679 & 0.79 & 4.18 & -1.21 & 1.2111 & 1.9891 \\
0.05 & 0 & -1.6005 & 0.88 & 2.84 & -1.12 & 1.1299 & 1.9679 \\
0.2 & 0 & -1.2467 & 0.99 & 1.42 & -1.01 & 1.0168 & 1.9417 \\
0.5 & 0 & -0.8778 & 1.11 & 0.80 & -0.71 & 0.8904 & 1.9172 \\
1.0 & 0 & -0.5000 & 1.26 & 0.43 & -0.32 & 0.7500 & 1.8958 \\
1.5 & 0 & -0.2266 & 1.36 & 0.25 & -0.16 & 0.6398 & 1.8832 \\
2.0 & $0(\log )$ & $0(\log )$ & 1.49 & 0.15 & -0.08 & 0.5417 & 1.8750 \\
2.5 & $0.26(1)$ & 0.2036 & 1.64 & 0.10 & 0.20 & 0.4474 & 1.8697 \\
3.0 & $0.45(1)$ & 0.4000 & 1.84 & 0.06 & 0.41 & 0.3500 & 1.8667 \\
3.5 & $0.636(2)$ & 0.6101 & 2.04 & 0.04 & 0.61 & 0.2375 & 1.8662 \\
\hline
\end{tabular}




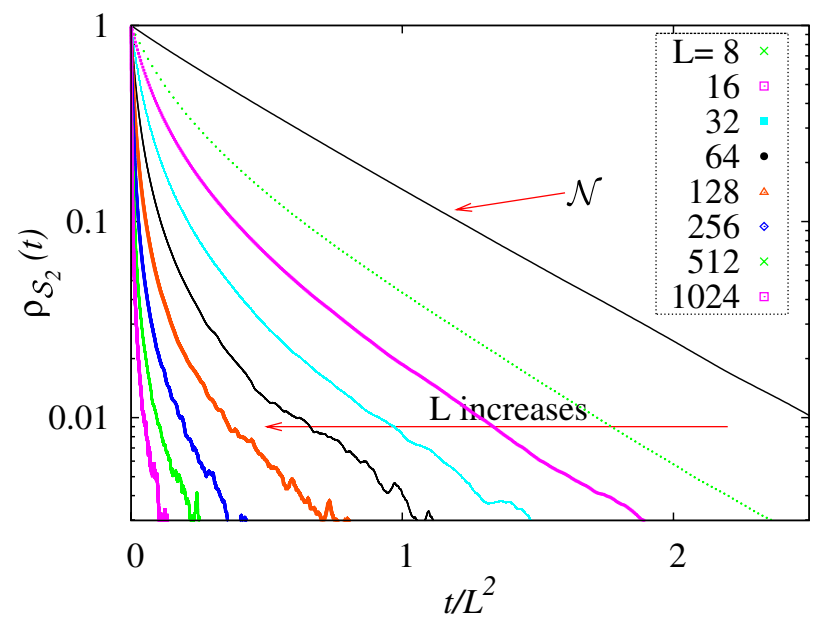

FIG. 3 (color online). Autocorrelation function $\rho_{\mathcal{S}_{2}}(t)$ vs $t / L^{2}$ for the critical two-dimensional random-cluster model at $q=$ 0.2 . The autocorrelation function $\rho_{\mathcal{N}}(t)$ is shown for comparison.

Finally we can analyze the universal crossover from short-time to long-time behavior, which we hypothesize is given by the "two-time-scale" Ansatz $\rho_{\mathcal{S}_{2}}(t)=$ $f\left(t / L^{w}\right) g\left(t / L^{d+z_{\exp }}\right)$ with $w<d+z_{\exp }$. By plotting $(1+$ $\left.a t / L^{w}\right)^{r} \rho_{\mathcal{S}_{2}}(t)$ versus $t / L^{d+z_{\exp }}$, a fairly clear scaling curve is seen (Fig. 5), though it is noisy for large lattices. Using this scaling Ansatz to compute the area under the curve of $\rho_{\mathcal{S}_{2}}(t)$, we conclude that

$$
z_{\mathrm{int}, \mathcal{S}_{2}}= \begin{cases}r(w-d)+(1-r) z_{\exp } & \text { if } r<1 \\ w-d & \text { if } r>1\end{cases}
$$

Similar analyses for the other values of $q$ yield the exponents reported in Table I. Note that critical speeding-up $(w<d)$ and critical slowing-down $\left(z_{\exp }>0\right)$ can coexist. For $q \lesssim 2.1$ we have critical speeding-up in the strong sense $\left(z_{\mathrm{int}, \mathcal{S}_{2}}<0\right)$.

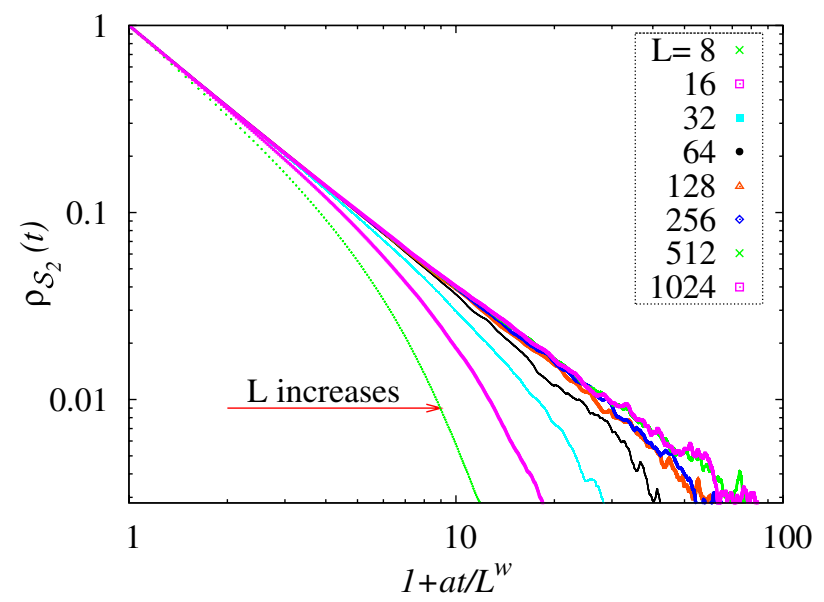

FIG. 4 (color online). Autocorrelation function $\rho_{\mathcal{S}_{2}}(t)$ vs $1+$ $a t / L^{w}$ for the critical two-dimensional random-cluster model at $q=0.2$, with $w=0.99$ and $a=0.55$.

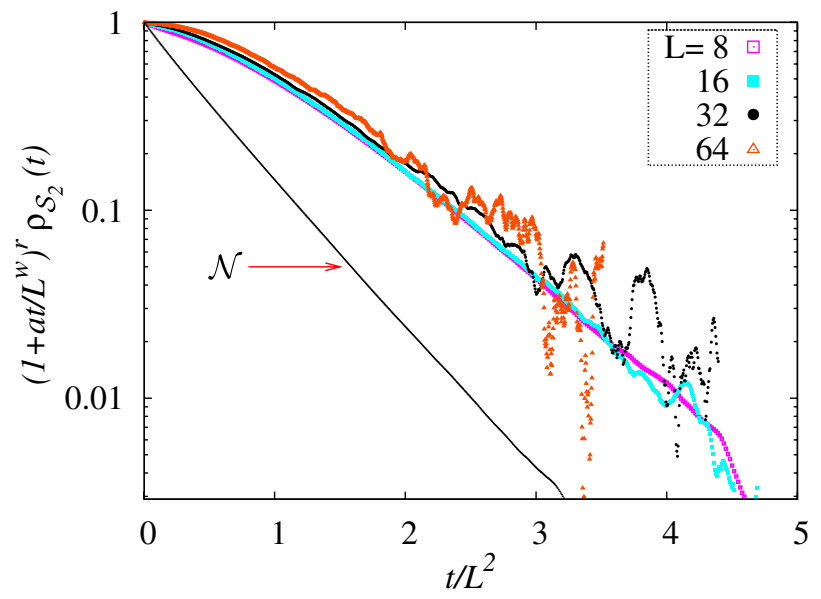

FIG. 5 (color online). $\quad\left(1+t / L^{w}\right)^{r} \rho_{\mathcal{S}_{2}}(t)$ vs $t / L^{2}$ for the critical two-dimensional random-cluster model at $q=0.2$, with $w=$ $0.99, a=0.55$, and $r=1.42$. The autocorrelation function $\rho_{\mathcal{N}}(t)$ is shown for comparison.

Critical speeding-up also occurs in the random-cluster model in dimensions $d>2$. We simulated the threedimensional random-cluster model [22] at the estimated critical points $w=v / q=0.43365$ for $q=0$ [19], $p=$ $v /(1+v)=0.2488126$ for $q=1$ [26], and $v=e^{2 \beta}-1$ with $\beta=0.22165455$ for $q=2$ [27], using lattice sizes $4 \leq L \leq 256$ for $q=0,1$ and $4 \leq L \leq 64$ for $q=2$. The qualitative behavior was the same as in $d=2$, and the estimated exponents are shown in Table II. Our value of $z_{\text {exp }}$ for $q=2$ is consistent with that of Wang, Kozan, and Swendsen [6]. In fact, $\tau_{\text {int, } \mathcal{N}} / C_{H}$ for $q=2$ is close to constant, so it is conceivable that $z_{\exp }=\alpha / \nu$ exactly.

In retrospect it is not surprising that a "global" observable like $\mathcal{S}_{2}$ could exhibit significant decorrelation in a time much less than one sweep. After all, FK clusters are fractals: a large cluster can sometimes be broken into two large pieces by one or a few bond deletions, and two large clusters can sometimes be joined by one or a few bond insertions. This reasoning suggests correctly that the critical speeding-up should be strongest when the cluster is most fragile, i.e., the red-bond fractal dimension $d_{\text {red }}$ [23] is largest, namely at small $q$.

We can pursue this idea further and suggest that the decorrelation of $\mathcal{S}_{2}$ is caused principally by hitting a few

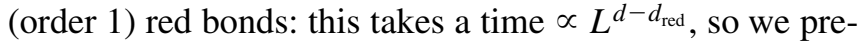
dict $w=d-d_{\text {red. }}$. Our data (Tables I and II) are in amazingly good agreement with this prediction for $q \lesssim 2$, i.e.,

TABLE II. Same as Table I, for the three-dimensional randomcluster model. $\alpha / \nu$ and $d_{F}$ from [19], [29], [27,28] for $q=0,1$, $2 ; d_{\text {red }}$ from [29], [30] for $q=1,2$.

\begin{tabular}{llrccccc}
\hline \hline$q$ & $z_{\exp }$ & $\alpha / \nu$ & $w$ & $r$ & $z_{\text {int } \mathcal{S}_{2}}$ & $d_{\text {red }}$ & $d_{F}$ \\
\hline 0 & 0 & $-1.44(5)$ & 1.52 & 1.04 & -1.48 & $?$ & $2.5838(5)$ \\
1 & 0 & $-0.713(1)$ & 1.87 & 0.32 & -0.36 & $1.1437(6)$ & $2.5219(2)$ \\
2 & $0.35(1)$ & $0.174(2)$ & 2.55 & 0.08 & 0.29 & $0.757(2)$ & $2.4816(1)$ \\
\hline \hline
\end{tabular}


when $z_{\exp }=0$. However, they deviate from it when $z_{\exp }>0$ for reasons that we do not yet understand. Note, in particular, that for $d \geq 6$ and $q=1$ (resp. $0 \leq q \leq 1$ ), we expect $d_{\text {red }}=y_{t 0}=2$ (resp. $\left.d_{\text {red }} \geq 2\right)$ and hence $w=$ $d-2$ (resp. $w \leq d-2$ ).

We lack, at present, any theory (or even any numerology) for $r$. But in two dimensions it seems that $r \rightarrow 5$ (resp. 0 ) as $q \rightarrow 0$ (resp. 4).

Let us note that a similar "two-time-scale" behavior is observed in the pivot dynamics [31] for ordinary random walk (or self-avoiding walk), in which "global" observables such as the end-to-end distance and the radius of gyration exhibit a fast relaxation $\tau_{\text {short }} \sim N^{0}$ while the slowest mode has $\tau_{\exp } \sim N$ (here $N$ is the number of steps in the walk). Indeed, it is conceivable that most types of dynamics - perhaps even single-spin-flip (Glauber) dynamics - exhibit this two-time-scale effect (i.e., $w<d+$ $z_{\exp }$ and hence $z_{\text {int, } \mathcal{O}}<z_{\text {exp }}$ ) to a greater or lesser extent.

On a practical level, our results show that the Sweeny algorithm is, despite its local nature, an unexpectedly efficient algorithm for simulating the random-cluster model. For $q \lesssim 1.5$ its efficiency is enhanced by strong critical speeding-up. Even for larger values of $q$, it is a potential competitor to the Chayes-Machta [20] cluster algorithm if efficient dynamic connectivity-checking algorithms can be found $[14,15]$.

Details of these simulations and their data analysis will be reported separately [15].

This work was supported in part by NSF Grants No. PHY-0116590 and No. PHY-0424082.

[1] P. C. Hohenberg and B. I. Halperin, Rev. Mod. Phys. 49, 435 (1977).

[2] Monte Carlo Methods in Statistical Physics, edited by K. Binder (Springer-Verlag, Berlin, 1986), 2nd ed.; Applications of the Monte Carlo Method in Statistical Physics, edited by K. Binder (Springer-Verlag, Berlin, 1987), 2nd ed.; The Monte Carlo Method in Condensed Matter Physics, edited by K. Binder (Springer-Verlag, Berlin, 1995), 2nd ed.

[3] A.D. Sokal, in Functional Integration: Basics and Applications, edited by C. de Witt-Morette, P. Cartier, and A. Folacci (Plenum, New York, 1997), pp. 131-192.

[4] P. W. Kasteleyn and C. M. Fortuin, J. Phys. Soc. Jpn. 26 (Suppl.), 11 (1969); C. M. Fortuin and P. W. Kasteleyn, Physica (Amsterdam) 57, 536 (1972).

[5] G. Grimmett, The Random-Cluster Model (SpringerVerlag, Berlin, 2006).

[6] M. Sweeny, Phys. Rev. B 27, 4445 (1983). For variants of the Sweeny algorithm, see F. Gliozzi, Phys. Rev. E 66, 016115 (2002) as corrected by J.-S. Wang, O. Kozan, and R. H. Swendsen, Phys. Rev. E 66, 057101 (2002).

[7] R. B. Potts, Proc. Cambridge Philos. Soc. 48, 106 (1952).

[8] F. Y. Wu, Rev. Mod. Phys. 54, 235 (1982); 55, 315(E) (1983); J. Appl. Phys. 55, 2421 (1984).
[9] P. Di Francesco, P. Mathieu, and D. Sénéchal, Conformal Field Theory (Springer-Verlag, Berlin, 1997).

[10] O. Schramm, Isr. J. Math. 118, 221 (2000); S. Rohde and O. Schramm, Ann. Math. 161, 883 (2005); G. F. Lawler, Conformally Invariant Processes in the Plane (American Mathematical Society, Providence, 2005).

[11] W. Kager and B. Nienhuis, J. Stat. Phys. 115, 1149 (2004); J. Cardy, Ann. Phys. (N.Y.) 318, 81 (2005).

[12] D. Stauffer and A. Aharony, Introduction to Percolation Theory (Taylor \& Francis, London, 1992), 2nd ed.

[13] This is the "heat-bath" version of the Sweeny dynamics, in which the new occupation state of the bond $e$ is independent of the old occupation state. A slightly more efficient alternative is the "Metropolis" version, in which a proposal to flip the occupation state of $e$ is accepted or rejected according to the Metropolis criterion.

[14] D. Alberts, G. Cattaneo, and G. F. Italiano, ACM J. Exp. Algorithmics 2, 5 (1997); M. R. Henzinger and V. King, J. ACM 46, 502 (1999); R. D. Iyer, Jr. et al., ACM J. Exp. Algorithmics 6, 4 (2001); J. Holm, K. de Lichtenberg, and M. Thorup, J. ACM 48, 723 (2001).

[15] Y. Deng, T. M. Garoni, and A. D. Sokal (to be published).

[16] R.J. Baxter, Exactly Solved Models in Statistical Mechanics (Academic, New York, 1982).

[17] B. Nienhuis, J. Stat. Phys. 34, 731 (1984).

[18] We stress that this is a lattice-dependent question; the answer is not universal among lattices of a given dimension $d$. For further discussion, see H. W. J. Blöte, Y. Deng, X. Qian, and A. D. Sokal (unpublished).

[19] Y. Deng, T. M. Garoni, and A. D. Sokal, Phys. Rev. Lett. 98, 030602 (2007).

[20] L. Chayes and J. Machta, Physica (Amsterdam) 254A, 477 (1998).

[21] X.-J. Li and A. D. Sokal, Phys. Rev. Lett. 63, 827 (1989), see last paragraph.

[22] We used the Metropolis version of the Sweeny algorithm: see [13] above.

[23] In infinite volume, the "red" bonds are those (backbone) bonds whose removal disconnects the infinite cluster into two infinite pieces. In $d=2$, the red-bond and wholecluster fractal dimensions and the specific-heat exponent are $d_{\text {red }}=(4-g)(4+3 g) / 8 g, d_{F}=y_{h 0}=(g+2)(g+$ 6) $/ 8 g$, and $\alpha / \nu=2 y_{t 0}-d=(4 g-12) / g$, where $q=$ $4 \cos ^{2}(\pi g / 4)$ and $2 \leq g \leq 4$. At $q=1$ in all dimensions, $d_{\text {red }}$ coincides with the thermal exponent $y_{t 0}$. See [17] and A. Coniglio, Phys. Rev. Lett. 62, 3054 (1989).

[24] For $q=2$ and 2.5 , the ratio $\tau_{\text {int, } \mathcal{N}} / C_{H}$ is decreasing in $L$, which suggests that $z_{\exp }=\alpha / \nu$ exactly. For $q=3$ and 3.5 , this ratio is very slowly increasing at large $L$.

[25] Y. Deng, T. M. Garoni, J. Machta, G. Ossola, M. Polin, and A. D. Sokal, arXiv:0705.2751.

[26] C. D. Lorenz and R. M. Ziff, Phys. Rev. E 57, 230 (1998).

[27] Y. Deng and H.W. J. Blöte, Phys. Rev. E 68, 036125 (2003).

[28] A. Pelissetto and E. Vicari, Phys. Rep. 368, 549 (2002).

[29] Y. Deng and H.W. J. Blöte, Phys. Rev. E 72, 016126 (2005).

[30] Y. Deng and H.W. J. Blöte, Phys. Rev. E 70, 056132 (2004).

[31] N. Madras and A. D. Sokal, J. Stat. Phys. 50, 109 (1988), see Section 3.3. 